\title{
Non-minimal coupling of torsion-matter satisfying null energy condition for wormhole solutions
}

\author{
Abdul Jawad ${ }^{\mathrm{a}}$, Shamaila Rani ${ }^{\mathrm{b}}$ \\ Department of Mathematics, COMSATS Institute of Information Technology, Lahore 54000, Pakistan
}

Received: 1 August 2016/ Accepted: 3 December 2016 / Published online: 22 December 2016

(c) The Author(s) 2016. This article is published with open access at Springerlink.com

\begin{abstract}
We explore wormhole solutions in a non-minimal torsion-matter coupled gravity by taking an explicit nonminimal coupling between the matter Lagrangian density and an arbitrary function of the torsion scalar. This coupling describes the transfer of energy and momentum between matter and torsion scalar terms. The violation of the null energy condition occurred through an effective energy-momentum tensor incorporating the torsion-matter non-minimal coupling, while normal matter is responsible for supporting the respective wormhole geometries. We consider the energy density in the form of non-monotonically decreasing function along with two types of models. The first model is analogous to the curvature-matter coupling scenario, that is, the torsion scalar with $T$-matter coupling, while the second one involves a quadratic torsion term. In both cases, we obtain wormhole solutions satisfying the null energy condition. Also, we find that the increasing value of the coupling constant minimizes or vanishes on the violation of the null energy condition through matter.
\end{abstract}

\section{Introduction}

The topological handles which connect distant regions of the universe as a bridge or tunnel is named a wormhole. The most amazing thing is the two-way travel through a wormhole tunnel which happened when the throat remains open that is, to prevent the wormhole from collapse at a non-zero minimum value of the radial coordinate. In order to keep the throat open, the exotic matter is used which violates the null energy condition and one elaborates the wormhole trajectories as hypothetical paths. The violation of the null energy

\footnotetext{
a e-mails: jawadab181@yahoo.com; abduljawad@ciitlahore.edu.pk

b e-mails: shamailatoor.math@yahoo.com; drshamailarani@ciitlahore. edu.pk
}

condition is the basic ingredient to integrate wormhole solutions. This configuration was first studied by Flamm [1] and then led Einstein and Rosen [2] to contribute the successive steps for the construction of wormhole solutions. The work of Morris and Thorne [3] evoked the wormhole scenario and led to new directions. The usual types of matter are considered to satisfy the energy conditions, therefore, some exotic type matter is employed for these solutions. There exist some wormhole solutions in semi-classical gravity through quantum effects such as Hawking evaporation and Casimir effects $[3,4]$ where the energy conditions are violated. One may take some such types of matter which acted as exotic matter, for instance, phantom energy [5-7], tachyon matter [8], generalized Chaplygin gas [9], some non-minimal kinetic coupling, etc.

In order to find some realistic sources that support the wormhole geometry or minimize the usage of exotic matter, different varieties of wormhole solutions are explored. These include thin-shell, dynamical, and rotating wormholes [10-16]. However, our concentration goes toward modified theories of gravity where the effective scenario gives the violation of the null energy condition and the matter source supports the wormholes. In $f(R)$ gravity, Lobo and Oliveira [17] found that the higher curvature terms in the effective energymomentum tensor are responsible for the necessary violation for the wormhole solutions. They assumed a particular the shape function along with various fluids to check the validity of energy conditions. Jamil et al. [18] discussed several static wormhole solutions in this version of gravity with a noncommutative geometry background through a Gaussian distribution. They were first to consider a power-law solution and construct the wormhole geometry as well as analyze the validity of the energy conditions. Second, they explored these solutions with the help of the shape function. Taking into account a Lorentzian distribution of the energy density, Rahaman et al. [19] derived some new exact solutions in the same manner and gave some viable wormhole solutions. 
In extended teleparallel gravity, being a modification of teleparallel gravity [20-46], static as well as dynamical wormhole solutions are also explored. In this way, Böhmer et al. [47] investigated wormhole solutions in this gravity by taking some specific $f(T)$ forms, the shape function as well as redshift functions which are the basic characteristics of these solutions. Assuming different fluids such as barotropic, isotropic, and anisotropic, Jamil et al. [48] explored the possibility of some realistic sources for wormhole solutions. Sharif and Rani explored the wormhole solutions in this gravity taking a noncommutative background with a Gaussian distribution [49], dynamical wormhole solutions [50], for the traceless fluid [51], with the inclusion of charge [52] and galactic halo scenario [53]. They considered some power-law $f(T)$ functions for which the effective energymomentum tensor depending on torsion contributed terms violating the null energy condition. Recently, Jawad and Rani [54] constructed wormhole solutions via a Lorentzian distribution in a noncommutative background. They concluded that there exists a possibility of some realistic wormhole solutions satisfying energy conditions and staying in equilibrium. We also studied some higher dimensional wormhole solutions in Einstein-Gauss-Bonnet gravity [55].

The modification of theories taking some non-minimal coupling between matter and curvature has become a center of interest nowadays. There exist such theories involving these couplings like $f(R)=f_{1}(R)+\left[1+\lambda f_{2}(R)\right] \mathcal{L}_{\mathrm{m}}$ and $f\left(R, \mathcal{L}_{\mathrm{m}}\right),[56,57]$ etc. Harko et al. [58] introduced the most general conditions in the framework of modified gravity, that is, the matter threading the wormhole throat satisfies all of the energy conditions, while the gravitational fluid (such as higher order curvature terms) supports the nonstandard wormhole geometries. They explicitly showed that wormhole geometries can be theoretically constructed without the presence of exotic matter but are sustained in the context of modified gravity. Taking into account some specific cases of modified theories of gravity, namely, $f(R)$ gravity, the curvature-matter coupling and the $f\left(R, \mathcal{L}_{\mathrm{m}}\right)$ generalization, they showed explicitly that one may choose the parameters of the theory such that the matter threading the wormhole throat satisfies the energy conditions.

Following the same scenario as for $f(R)$ theory, Harko et al. [59] proposed a non-minimal torsion-matter coupling as a extension of $f(T)$ gravity. In this gravity, two arbitrary functions $f_{1}(T)$ and $f_{2}(T)$ are introduced such as $f_{1}$ is the extension of geometric part while $f_{2}$ is coupled with matter Lagrangian part through some coupling constant. They discussed this theory as regards cosmological aspects of an evolving universe and deduced that the universe may represent quintessence, phantom or crossing of phantom-divide line, inflationary era, de Sitter accelerating phase, in short, one has a unified description. In this version of gravity, Nashed [60], Feng et al. [61], and Carloni et al. [62] studied spherically symmetric solutions, cosmological evolutions, and they compared their results with observational data and phase space analysis, respectively. Garcia and Lobo [63] explored wormhole solutions by a non-minimal curvaturematter coupling taking linear functions, $f_{1}=R, f_{2}=R$. They concluded that the wormhole solutions in a realistic manner depend on higher values of the coupling parameter.

The paper has the following format. In Sect. 2, we describe the $f(T)$ gravity and a non-minimal torsion-matter coupling extension. Section 3 is devoted to the gravitational field equations for wormhole geometry in the coupling scenario. We find the general conditions on the matter part for the validity of the null energy condition. Also, we examine the effective energy-momentum tensor being responsible for the violation of the null energy condition. In Sect. 4, we explore wormhole solutions taking into account two well-known models. These models involving a linear torsion scalar coupled with $T$-matter and quadratic torsion term with matter representing a wormhole geometry. In the last section, we summarize the results.

\section{Non-minimal Torsion-matter Coupling}

In this section, we mainly review the torsion-based gravitational paradigm. The torsional scenario begins with a spacetime undergoing the absolute parallelism where the parallel vector field $h_{\beta}{ }^{a}$ determines

$\Gamma_{b c}^{a}=h_{\beta}^{a} h_{b, c}^{\beta}$,

which is a non-symmetric affine connection with vanishing curvature and $h_{\beta a, b}=\partial_{b} h_{\beta a}$. Here we apply Latin indices for the notation for a tangent spacetime, while coordinate spacetime indices are presented by Greek letters. The parallel vector fields (vierbein or tetrad fields) are the basic dynamical variables from which one deduces an orthonormal basis for the tangent space, i.e., $\mathbf{h}_{a} \cdot \mathbf{h}_{b}=\eta_{a b}$ where $\eta=\operatorname{diag}(1,-1,-1,-1)$. In terms of vierbein components, $h^{\beta}$, the vierbein fields are defined as $\mathbf{h}_{a}=h^{\beta}{ }_{a} \partial_{\beta}$, while the inverse components $h_{a}{ }^{\beta}$ meet the following conditions:

$h^{a}{ }_{\beta} h_{b}{ }^{\beta}=\delta_{b}^{a}, \quad h_{a}{ }^{\beta} h^{a}{ }_{\alpha}=\delta_{\alpha}^{\beta}$.

The metric tensor is obtained through the relation $g_{\beta \alpha}=$ $\eta_{a b} h^{a}{ }_{\beta} h_{\alpha}^{b}$, which gives the metric determinant as $\sqrt{-g}=$ $e=\operatorname{det}\left(h_{\beta}^{a}\right)$. Using Eq. (1), the antisymmetric part of Weitzenböck connection yields

$T_{\alpha \sigma}^{\beta}=\widetilde{\Gamma}_{\sigma \alpha}^{\beta}-\widetilde{\Gamma}_{\alpha \sigma}^{\beta}=h_{a}^{\beta}\left(\partial_{\sigma} h_{\alpha}^{a}-\partial_{\alpha} h_{\sigma}^{a}\right)$,

where $T^{\beta}{ }_{\alpha \gamma}=-T^{\beta}{ }_{\gamma \alpha}$, i.e., it is antisymmetric in its lower indices. It is noted that under the parallel transportation of the 
vierbein field, the curvature of the Weitzenböck connection vanishes. Using this tensor, we obtain the contorsion tensor: $K_{\beta}^{\alpha \gamma}=-\frac{1}{2}\left(T_{\beta}^{\alpha \gamma}-T_{\beta}^{\gamma \alpha}-T_{\beta}^{\alpha \gamma}\right)$ and the superpotential tensor: $S_{\beta}{ }^{\alpha \gamma}=\frac{1}{2}\left[\delta_{\beta}^{\alpha} T^{\mu \gamma}{ }_{\mu}-\delta_{\beta}^{\gamma} T^{\mu \alpha}{ }_{\mu}+K_{\beta}^{\alpha \gamma}\right]$. The torsion scalar takes the form

$T=S_{\beta}{ }^{\alpha \gamma} T^{\beta}{ }_{\alpha \gamma}$.

The action of $f(T)$ gravity is given by [20-46]

$\mathcal{S}=\frac{1}{16 \pi \mathcal{G}} \int e\left\{f(T)+\mathcal{L}_{\mathrm{m}}\right\} d^{4} x$

$\mathcal{G}$ is the gravitational constant and $f$ represents the generic differentiable function of the torsion scalar describing an extension of the teleparallel gravity. The term $\mathcal{L}_{\mathrm{m}}$ describes the matter part of the action such as $\mathcal{L}_{\mathrm{m}}=\mathcal{L}_{\mathrm{m}}(\rho, p)$ where $\rho, p$ are the energy density and pressure with the null energy, while we neglect the radiation section for the sake of simplicity. By the variation of this action w.r.t. vierbein field, the following field equations result:

$$
\begin{aligned}
& h_{a}{ }^{\beta} S_{\beta}{ }^{\alpha \sigma} \partial_{\alpha} T f_{T T}+\left[h_{a}{ }^{\beta} T^{\lambda}{ }_{\alpha \beta} S_{\lambda}{ }^{\sigma \alpha}+\frac{1}{e} \partial_{\alpha}\left(h h_{a}{ }^{\beta} S_{\beta}{ }^{\alpha \sigma}\right)\right] \\
& \times f_{T}+\frac{1}{4} h_{a}{ }^{\sigma} f=4 \pi \mathcal{G} h_{a}{ }^{\beta} \Theta_{\beta}^{\sigma},
\end{aligned}
$$

where the subscripts involving $T$ and $T T$ represent first and second order derivatives of $f$ with respect to $T$, respectively. For the sake of simplicity, we assume $8 \pi \mathcal{G}=1$ in the following.

The $f(T)$ field equations in terms of Einstein tensor gain a remarkable importance in order to discuss various cosmological and astrophysical scenarios [20-46]. This type of field equations occurs by replacing partial derivatives with covariant derivatives along with the compatibility of metric tensor, i.e., $\nabla_{\sigma} g_{\beta \alpha}=0$. Using the relations $T^{\mu(\nu \gamma)}=K^{(\mu \nu) \gamma}=$ $S^{\mu(\nu \gamma)}=0$, the torsion, contorsion, and superpotential tensor become

$$
\begin{aligned}
T_{\beta \alpha}^{\sigma} & =h_{a}^{\sigma}\left(\nabla_{\beta} h_{\alpha}^{a}-\nabla_{\alpha} h_{\beta}^{a}\right), \quad K_{\beta \alpha}^{\sigma}=h_{a}^{\sigma} \nabla_{\alpha} h_{\beta}^{a}, \\
S_{\sigma}{ }^{\alpha \beta} & =\eta^{a b} h_{a}^{\beta} \nabla_{\sigma} h_{b}^{\alpha}+\delta_{\sigma}^{\alpha} \eta^{a b} h_{a}^{\tau} \nabla_{\tau} h_{b}^{\beta}-\delta_{\sigma}^{\beta} \eta^{a b} h_{a}^{\tau} \nabla_{\tau} h_{b}^{\alpha} .
\end{aligned}
$$

The curvature tensor referred to the Weitzenböck connection vanishes, while the Riemann tensor related with the LeviCivita connection $\Gamma_{\mu \nu}^{\gamma}$ is given by

$R_{\beta \lambda \alpha}^{\sigma}=\nabla_{\alpha} K_{\beta \lambda}^{\sigma}-\nabla_{\lambda} K_{\beta \alpha}^{\sigma}+K_{\tau \alpha}^{\sigma} K_{\beta \lambda}^{\tau}-K^{\sigma}{ }_{\tau \lambda} K_{\beta \alpha}^{\tau}$.

We obtain the Ricci tensor and scalar as follows:

$$
\begin{aligned}
R_{\beta \alpha} & =-\nabla^{\sigma} S_{\alpha \sigma \beta}-g_{\beta \alpha} \nabla^{\sigma} T_{\sigma \lambda}^{\lambda}-S_{\beta}^{\sigma \lambda} K_{\lambda \sigma \alpha}, \\
R+T & =-2 \nabla^{\sigma} T_{\sigma \alpha}^{\alpha},
\end{aligned}
$$

where we use $S_{\sigma \alpha}^{\alpha}=-2 T^{\alpha}{ }_{\sigma \alpha}=2 K^{\alpha}{ }_{\sigma \alpha}$. Substituting Eq. (6) along with Einstein tensor $G_{\beta \alpha}=R_{\beta \alpha}-\frac{1}{2} g_{\beta \alpha} R$, we get

$G_{\beta \alpha}-\frac{1}{2} g_{\beta \alpha} T=-\nabla^{\sigma} S_{\alpha \sigma \beta}-S_{\beta}^{\tau \sigma} K_{\sigma \tau \alpha}$.

Finally, inserting this equation in Eq. (5), it yields

$f_{T} G_{\beta \alpha}+\frac{1}{2} g_{\beta \alpha}\left(f-T f_{T}\right)+Z_{\beta \alpha} f_{T T}=\Theta_{\beta \alpha}$,

where $Z_{\beta \alpha}=S_{\alpha \beta}{ }^{\sigma} \nabla_{\sigma} T$. This equation expresses a similar structure to $f(R)$ gravity at least up to equation level and representing GR for the limit $f(T)=T$. We take trace of the above equation, i.e., $Z f_{T T}-(R+2 T) f_{T}+2 f=\Theta$, with $Z=Z^{\alpha}{ }_{\alpha}$ and $\Theta=\Theta^{\alpha}{ }_{\alpha}$ to simplify the field equations. The $f(T)$ field equations can be rewritten as

$G_{\beta \alpha}=\frac{1}{f_{T}}\left[\Theta_{\beta \alpha}^{m}-Z_{\beta \alpha} f_{T T}-\frac{1}{2}\left(f-T f_{T}\right)\right]$,

where $\Theta_{\beta \alpha}^{m}$ is the energy-momentum tensor corresponding to matter Lagrangian.

Taking into account a non-minimal coupling between torsion and matter, Harko et al. [59] defined the action as follows:

$\mathcal{S}=\int e\left\{f_{1}+\left(1+\omega f_{2}\right) \mathcal{L}_{\mathrm{m}}\right\} d^{4} x$,

where $\omega$ is the coupling constant having units of mass ${ }^{-2}$ and $f_{1}, f_{2}$ are arbitrary differential functions of torsion scalar. Applying the tetrad variation on this action, we obtain the following set of equations:

$$
\begin{aligned}
h_{a}{ }^{\beta} & S_{\beta}{ }^{\alpha \sigma} \partial_{\alpha} T\left(f_{1}^{\prime \prime}+\omega f_{2}^{\prime \prime} \mathcal{L}_{\mathrm{m}}\right)+\left[h_{a}{ }^{\beta} T^{\lambda}{ }_{\alpha \beta} S_{\lambda}{ }^{\sigma \alpha}\right. \\
& \left.+\frac{1}{e} \partial_{\alpha}\left(h h_{a}{ }^{\beta} S_{\beta}{ }^{\alpha \sigma}\right)\right]\left(f_{1}^{\prime}+\omega f_{2}^{\prime} \mathcal{L}_{\mathrm{m}}\right) \\
& +\frac{1}{4} h_{a}{ }^{\sigma} f_{1}-\frac{1}{4} \omega \partial_{\alpha} T h_{a}{ }^{\beta} \mathcal{T}_{\beta}^{\sigma \alpha} f_{2}^{\prime}+\omega f_{2}^{\prime} h_{a}{ }^{\beta} S_{\beta}{ }^{\sigma \alpha} \partial_{\alpha} \mathcal{L}_{\mathrm{m}} \\
= & \frac{1}{2}\left(1+\omega f_{2}\right) h_{a}{ }^{\beta} \Theta_{\beta}^{\sigma},
\end{aligned}
$$

where the number of primes denotes the correspondingly order derivative with respect to the torsion scalar, and $\mathcal{T}_{a}^{\sigma \alpha}=$ $\frac{\partial \mathcal{L}_{\mathrm{m}}}{\partial \partial_{\alpha} h^{a} \sigma}$. We assume here that the matter Lagrangian $\mathcal{L}_{\mathrm{m}}$ is independent of derivatives of the tetrad, which results in the vanishing of $\mathcal{T}_{a}^{\sigma \alpha}$. Also, we have the Bianchi identities of teleparallel gravity:

$$
\begin{aligned}
\nabla_{\beta} \Theta_{\tau}^{\beta}= & \frac{4}{1+\omega f_{2}} K^{\lambda}{ }_{\beta \tau} S_{\lambda}^{\beta \alpha} \nabla_{\alpha}\left(f_{1}^{\prime}+\omega f_{2}^{\prime} \mathcal{L}_{\mathrm{m}}\right) \\
& -\frac{\omega f_{2}^{\prime}}{1+\omega f_{2}}\left(\Theta_{\tau}^{\beta}-\mathcal{L}_{\mathrm{m}} \delta_{\tau}^{\beta}\right) \nabla_{\beta} T .
\end{aligned}
$$

This equation represents the substitute of energy and momentum between torsion and matter through the defined coupling. 
The $f(T)$ field equations for the torsion-matter coupling in the form of the Einstein tensor are given by

$$
\begin{aligned}
G_{\beta \alpha}= & \frac{1}{f_{1 T}+\omega f_{2 T} \mathcal{L}_{\mathrm{m}}} \\
& \times\left[\Theta_{\beta \alpha}^{+}-S_{\alpha \beta}{ }^{\lambda} \nabla_{\lambda}\left(f_{1 T T}+\omega f_{2 T T} \mathcal{L}_{\mathrm{m}}\right)-\mathcal{J} g_{\beta \alpha}\right]
\end{aligned}
$$

where

$$
\begin{aligned}
\mathcal{J} & =\frac{1}{2}\left\{f_{1}-T\left(f_{1 T}+\omega f_{2 T} \mathcal{L}_{\mathrm{m}}\right)\right\}, \\
\Theta_{\beta \alpha}^{+} & =\left(1+\omega f_{2}\right) \Theta_{\beta \alpha}^{m}-\omega f_{2 T} S_{\alpha \beta}{ }^{\lambda} \nabla_{\lambda} \mathcal{L}_{\mathrm{m}} .
\end{aligned}
$$

It is noted that the matter Lagrangian density needs to be properly defined in the analysis of torsion-matter coupling. In the literature for curvature-matter coupling, the proposals for this matter Lagrangian density are as follows: [63].

(i) $\mathcal{L}_{\mathrm{m}}=p$, which reproduces the equation of state for a perfect fluid and proved as non-unique.

(ii) $\mathcal{L}_{\mathrm{m}}=-n e$ where $e$ denotes physical free energy defined by $e=-\mathbb{T} s+\frac{\rho}{n}, \mathbb{T}$ is the temperature, $s$ being the entropy of one particle and $n$ gives the particle number density.

(iii) $\mathcal{L}_{\mathrm{m}}=-\rho$ representing a natural choice which gives the energy in a local rest frame for the fluid.

\section{Gravitational field equations for wormhole geometry}

In order to construct the gravitational field equations in the framework of torsion-matter coupling, we first of all describe the wormhole geometry. Let us assume the wormhole metric as follows [49-54]:

$\mathrm{d} s^{2}=e^{2 \Psi(r)} \mathrm{d} t^{2}-\frac{1}{1-\frac{b}{r}} \mathrm{~d} r^{2}-r^{2} \mathrm{~d} \phi^{2}-r^{2} \sin ^{2} \phi \mathrm{d} \psi^{2}$,

where redshift function $\Psi$ and shape function $b$ are $r$ dependent functions. In order to set the geometry of the wormhole scenario, some constraints are required on both of these functions. These are described as follows.

- Shape function: The shape of the wormhole consists of two open mouths (two asymptomatically flat regions) in different regions of the space connected through the throat which is minimum non-zero value of the radial coordinate. This shape is maintained through the shape function $b(r)$ with increasing behavior and having ratio 1 with $r$. At the throat, we must have $b\left(r_{0}\right)=r_{0}$ as well as $1-\frac{b(r)}{r} \geq 0$. The flare-out condition being the fundamental property of wormhole geometry is defined as $\frac{1}{b^{2}}\left(b-b^{\prime} r\right)>0$

There is another constraint on the derivative of the shape function at the throat, i.e., $b^{\prime}\left(r_{0}\right)<1$, which must be fulfilled.

- Redshift function: The main purpose of wormholes is to give a way to have two-way traveling through its tunnel, which basically depends on the non-zero minimum value of $r$ at the throat. For this purpose, i.e., to keep the throat open, the redshift function plays its role by maintaining the no-horizon case at the throat. For this condition to hold, $\Psi$ must remain finite throughout the spacetime. This function calculates the gravitational redshift of a light particle. When this particle moves from a potential well to escape to infinity, there appears a reduction in its frequency, which is called gravitational redshift. At a particular value of $r$, its infinitely negative value expresses an event horizon at the throat. To prevent this situation of a horizon appearing so that the wormhole solution may provide a traversable way, the magnitude of its redshift function must be finite. This may be taken as $\Psi=0$, which gives $e^{2 \Psi(r)} \rightarrow 1$.

- Exotic matter: The existence of wormhole solutions requires some unusual type of matter, called exotic matter having negative pressure to violate the null energy condition. Thus, it becomes the basic factor for wormhole construction as the known classical forms of matter satisfying this condition. The search for such a source which provides the necessary violation with matter content obeying the null energy condition occupy a vast range of study in astrophysics.

The energy conditions originates through the Raychaudhuri equation in the realm of general relativity for the expansion regarding positivity of the term $R_{\beta \alpha} \mathcal{K}^{\beta} \mathcal{K}^{\alpha}$ where $\mathcal{K}^{\beta}$ denotes the null vector. This positivity guarantees a finite value of the parameter marking points on the geodesics directed by geodesic congruences. In terms of energymomentum tensor, the above term under positivity condition become $R_{\beta \alpha} \mathcal{K}^{\beta} \mathcal{K}^{\alpha}=\Theta_{\beta \alpha} \mathcal{K}^{\beta} \mathcal{K}^{\alpha} \geq 0$ in the framework of general relativity. However, in modified theories of gravity, we carry effective energy-momentum tensor which is further used in the above expression to study energy conditions. For instance, in the underlying case, Eq. (13) takes the form

$G_{\beta \alpha}=\Theta_{\beta \alpha}^{\mathrm{eff}}$, where

$\Theta_{\beta \alpha}^{\mathrm{eff}}=\frac{1}{f_{1 T}+\omega f_{2 T} \mathcal{L}_{\mathrm{m}}}\left[\Theta_{\beta \alpha}^{+}+\Theta_{\beta \alpha}^{\mathrm{TM}}\right]$,

where $\Theta_{\beta \alpha}^{\mathrm{TM}}=-S_{\alpha \beta}{ }^{\lambda} \nabla_{\lambda}\left(f_{1 T T}+\omega f_{2 T T} \mathcal{L}_{\mathrm{m}}\right)-\mathcal{J} g_{\beta \alpha}$ representing the contribution of torsion-matter coupling in the extended teleparallel gravity. The corresponding energy condition becomes $R_{\beta \alpha} \mathcal{K}^{\beta} \mathcal{K}^{\alpha}=\Theta_{\beta \alpha}^{\text {eff }} \mathcal{K}^{\beta} \mathcal{K}^{\alpha} \geq 0$, which is 
called the null energy condition. Taking into account a perfect fluid with the representation $\Theta_{\beta \alpha}=\left(\rho^{\text {eff }}+p^{\text {eff }}\right) \mathcal{U}_{\beta} \mathcal{U}_{\alpha}-$ $p^{\text {eff }} g_{\beta \alpha}$, this condition yields $\rho^{\text {eff }}+p^{\text {eff }} \geq 0$.

At this stage, we may impose a condition on the energymomentum tensor corresponding to a matter part such that $\Theta_{\beta \alpha}^{m} \mathcal{K}^{\beta} \mathcal{K}^{\alpha} \geq 0$ to thread the wormhole while $\Theta_{\beta \alpha}^{\text {eff }} \mathcal{K}^{\beta} \mathcal{K}^{\alpha} \leq 0$ gives the necessary violation. This condition implies the positivity of energy density in all local frames of references. Thus, it is important to study the constraints on $\Theta_{\beta \alpha}^{m}$ in order to form wormholes. Considering the violation of the null energy condition and Eq. (16), we get

$$
\begin{aligned}
& \frac{1}{f_{1 T}+\omega f_{2 T} \mathcal{L}_{\mathrm{m}}}\left[\left(1+\omega f_{2}\right) \Theta_{\beta \alpha}^{m}-\omega f_{2 T} S_{\alpha \beta}{ }^{\lambda} \nabla_{\lambda} \mathcal{L}_{\mathrm{m}}\right. \\
& \left.-S_{\alpha \beta}{ }^{\lambda} \nabla_{\lambda}\left(f_{1 T T}+\omega f_{2 T T} \mathcal{L}_{\mathrm{m}}\right)-\mathcal{J} g_{\beta \alpha}\right] \mathcal{K}^{\beta} \mathcal{K}^{\alpha}<0 .
\end{aligned}
$$

For the viable wormhole solutions, if $\left(f_{1 T}+\omega f_{2 T} \mathcal{L}_{\mathrm{m}}\right)>0$, then we obtain the following constraint:

$$
\begin{aligned}
0 & \leq \Theta_{\beta \alpha}^{m} \mathcal{K}^{\beta} \mathcal{K}^{\alpha}<\frac{1}{1+\omega f_{2}}\left[\omega f_{2 T} S_{\alpha \beta}{ }^{\lambda} \nabla_{\lambda} \mathcal{L}_{\mathrm{m}}\right. \\
& \left.+S_{\alpha \beta}{ }^{\lambda} \nabla_{\lambda}\left(f_{1 T T}+\omega f_{2 T T} \mathcal{L}_{\mathrm{m}}\right)+\mathcal{J} g_{\beta \alpha}\right] \mathcal{K}^{\beta} \mathcal{K}^{\alpha},
\end{aligned}
$$

where $1+\omega f_{2}>0$ must hold. For the case $\left(f_{1 T}+\omega f_{2 T} \mathcal{L}_{\mathrm{m}}\right)<$ 0 , the null energy condition straightforwardly gives

$$
\begin{aligned}
& \Theta_{\beta \alpha}^{m} \mathcal{K}^{\beta} \mathcal{K}^{\alpha}>\frac{1}{1+\omega f_{2}}\left[\omega f_{2 T} S_{\alpha \beta}{ }^{\lambda} \nabla_{\lambda} \mathcal{L}_{\mathrm{m}}\right. \\
& \left.+S_{\alpha \beta}{ }^{\lambda} \nabla_{\lambda}\left(f_{1 T T}+\omega f_{2 T T} \mathcal{L}_{\mathrm{m}}\right)+\mathcal{J} g_{\beta \alpha}\right] \mathcal{K}^{\beta} \mathcal{K}^{\alpha} .
\end{aligned}
$$

We consider the case $\mathcal{L}_{\mathrm{m}}=-\rho$ with no horizon condition, that is, $\Psi=0$, to construct the background geometry for wormhole solutions in the framework of $f(T)$ gravity having torsion-matter coupling. We take an anisotropic distribution of the fluid having energy-momentum tensor:

$$
\Theta_{\beta \alpha}^{m}=\left(\rho+p_{\mathrm{r}}\right) \mathcal{U}_{\beta} \mathcal{U}_{\alpha}-p_{\mathrm{r}} g_{\beta \alpha}+\left(p_{\mathrm{t}}-p_{\mathrm{r}}\right) \chi_{\beta} \chi_{\alpha},
$$

where $p_{\mathrm{r}}$ is the radial directed pressure component and $p_{\mathrm{t}}$ denotes tangential pressure component with $\rho=\rho(r), p=$ $p(r)$ satisfying $\mathcal{U}^{\beta} \mathcal{U}_{\alpha}=-\chi^{\beta} \chi_{\alpha}=1$. Taking into account Eqs. (13) and (14), we obtain a set of field equations as follows:

$$
\begin{aligned}
\frac{b^{\prime}}{r^{2}}= & \frac{1}{f_{1 T}-\omega f_{2 T} \rho} \\
& \times\left[\left(1+\omega f_{2}\right) \rho+\frac{\omega f_{2 T} \rho^{\prime}}{r}\left(1-\frac{b}{r}-\sqrt{1-\frac{b}{r}}\right)\right] \\
& -\frac{T^{\prime}\left(f_{1 T T}-\omega f_{2 T T} \rho\right)}{r\left(f_{1 T}-\omega f_{2 T} \rho\right)}\left(1-\frac{b}{r}-\sqrt{1-\frac{b}{r}}\right) \\
& -\frac{\mathcal{J}}{f_{1 T}-\omega f_{2 T} \rho}
\end{aligned}
$$

$$
\begin{aligned}
-\frac{b}{r^{3}}= & \frac{\left(1+\omega f_{2}\right) p_{\mathrm{r}}}{f_{1 T}-\omega f_{2 T} \rho}+\frac{\mathcal{J}}{f_{1 T}-\omega f_{2 T} \rho}, \\
-\frac{b^{\prime} r-b}{2 r^{3}}= & \frac{1}{f_{1 T}-\omega f_{2 T} \rho} \\
& \times\left[\left(1+\omega f_{2}\right) p_{\mathrm{t}}-\frac{\omega f_{2 T} \rho^{\prime}}{2 r}\left(1-\frac{b}{r}-\sqrt{1-\frac{b}{r}}\right)\right] \\
& +\frac{T^{\prime}\left(f_{1 T T}-\omega f_{2 T T} \rho\right)}{2 r\left(f_{1 T}-\omega f_{2 T} \rho\right)}\left(1-\frac{b}{r}-\sqrt{1-\frac{b}{r}}\right) \\
& +\frac{\mathcal{J}}{f_{1 T}-\omega f_{2 T} \rho},
\end{aligned}
$$

where a prime represents a derivative with respect to $r$ and

$T=\frac{2}{r^{2}}\left[2\left(1-\sqrt{1-\frac{b}{r}}\right)-\frac{b}{r}\right]$

The violation of the null energy condition $\left(\Theta_{\beta \alpha}^{\mathrm{eff}} \mathcal{K}^{\beta} \mathcal{K}^{\alpha}<\right.$ 0 ) for the field equations (18)-(20) is checked through the consideration of the radial null vector which yields

$$
\begin{aligned}
\rho^{\mathrm{eff}}+p_{\mathrm{r}}^{\mathrm{eff}}= & \frac{1}{f_{1 T}-\omega f_{2 T} \rho}\left[\left(1+\omega f_{2}\right)\left(\rho+p_{\mathrm{r}}\right)+\frac{1}{r}\left\{\omega f_{2 T} \rho^{\prime}\right.\right. \\
& \left.\left.-T^{\prime}\left(f_{1 T T}-\omega f_{2 T T} \rho\right)\right\}\left(1-\frac{b}{r}-\sqrt{1-\frac{b}{r}}\right)\right] \\
= & \frac{b^{\prime} r-b}{r^{3}}<0 \\
\Rightarrow & \rho^{\mathrm{eff}}+p_{\mathrm{r}}^{\mathrm{eff}}<0
\end{aligned}
$$

where the inequality comes through the flaring out condition of the shape function. In order to discuss the above scenario at the throat, we obtain the following relationship:

$$
\begin{aligned}
\rho^{\mathrm{eff}}\left(r_{0}\right)+p_{\mathrm{r}}^{\mathrm{eff}}\left(r_{0}\right)= & \frac{1}{f_{1 T_{0}}-\omega f_{2 T_{0}} \rho_{0}} \\
& \times\left[\left(1+\omega f_{2}\left(r_{0}\right)\right)\left(\rho_{0}+p_{r 0}\right)\right. \\
& +\frac{1}{r_{0}}\left\{\omega f_{2 T_{0}} \rho_{0}^{\prime}-T_{0}^{\prime}\left(f_{1 T_{0} T_{0}}-\omega f_{2 T_{0} T_{0}} \rho_{0}\right)\right\} \\
& \left.\times\left(1-\frac{b_{0}}{r_{0}}-\sqrt{1-\frac{b_{0}}{r_{0}}}\right)\right],
\end{aligned}
$$

which must satisfy the following constraint in order to meet the above inequality, that is:

$$
\begin{aligned}
(1+ & \left.\omega f_{2}\left(r_{0}\right)\right)\left(\rho_{0}+p_{r 0}\right) \\
& +\frac{1}{r_{0}} \omega f_{2 T_{0}} \rho_{0}^{\prime}\left(1-\frac{b_{0}}{r_{0}}-\sqrt{1-\frac{b_{0}}{r_{0}}}\right) \\
< & \frac{T_{0}^{\prime}}{r_{0}}\left(f_{1 T_{0} T_{0}}-\omega f_{2 T_{0} T_{0}} \rho_{0}\right)\left(1-\frac{b_{0}}{r_{0}}-\sqrt{1-\frac{b_{0}}{r_{0}}}\right),
\end{aligned}
$$

where $f_{1 T_{0}}-\omega f_{2 T_{0}} \rho_{0}>0$ while the inequality reverses if $f_{1 T_{0}}-\omega f_{2 T_{0}} \rho_{0}<0$. In order to write final field equa- 
tions in terms of the matter component, we observe that the last term in all Eqs. (18)-(20) change its sign using signature $(-,+,+,+)$ for wormhole spacetime while the remaining terms stay the same. This leads to the vanishing of last terms in each equation as energy density is independent of signature. Finally, the field equations can be rewritten as

$$
\begin{aligned}
b^{\prime}\left(f_{1 T}-\omega f_{2 T} \rho\right)= & r^{2}\left(1+\omega f_{2}\right) \rho+\omega \\
& \times\left[\omega f_{2 T} \rho^{\prime}-T^{\prime}\left(f_{1 T T}-\omega f_{2 T T} \rho\right)\right] \\
& \times\left(1-\frac{b}{r}-\sqrt{1-\frac{b}{r}}\right), \\
p_{\mathrm{r}}= & -\frac{b}{r^{3}} \frac{f_{1 T}-\omega f_{2 T} \rho}{1+\omega f_{2}}, \\
p_{\mathrm{t}}= & \frac{1}{2 r\left(1+\omega f_{2}\right)}\left[\left(f_{1 T}-\omega f_{2 T} \rho\right)\left(\frac{b}{r^{2}}-\frac{b^{\prime}}{r}\right)\right. \\
& -\left\{\omega \rho^{\prime} f_{2 T}+T^{\prime}\left(f_{1 T T}-\omega f_{2 T T} \rho\right)\right\} \\
& \left.\times\left(1-\frac{b}{r}-\sqrt{1-\frac{b}{r}}\right)\right] .
\end{aligned}
$$

\section{Wormhole solutions}

In order to discuss wormhole solutions, we have to deal with un-closed system of equations such that the three equations with $f_{1}, f_{2}, \rho, p_{\mathrm{r}}, p_{\mathrm{t}}$, and $b$ are unknown functions. Due to the non-linear appearance of these equations, the explicit functions are extremely difficult. However, we may adopt some alternative strategies keeping in mind the characteristics of wormhole geometry. To involve the effects of a non-minimal torsion-matter coupling to construct the wormhole solutions, we have to choose some viable models $f_{1}$ and $f_{2}$. Now we are left with four unknowns for which we may assume some kind of equation of state like an equation obeying the traceless fluid condition, a particular value of the shape function obeying all corresponding conditions or some type of energy density like density of static spherically symmetric smearing object, etc. We adopt the last approach of considering the energy density with the particular form [63]

$\rho=\rho_{0}\left(\frac{r_{0}}{r}\right)^{\sigma}$,

where $\rho_{0}$ and $\sigma$ are positive constants.

In the following, we consider two viable $f(T)$ models and study the different conditions of the shape functions as well as the null energy condition.

\subsection{Model 1}

As a first model, we consider the models $f_{1}=T$ and $f_{2}=$ $T$ analogous to the case of the curvature-matter coupling scenario where these models are taken as $f_{1}=R=f_{2}$ [63]. Substituting these values of the models along with Eqs. (25) in (22), we obtain the following differential equation for the shape function:

$$
\begin{aligned}
& b^{\prime}-\frac{\omega \sigma \rho_{0}\left(\frac{r_{0}}{r}\right)^{\sigma}}{1-\omega \rho_{0}\left(\frac{r_{0}}{r}\right)^{\sigma}}\left(\frac{b}{r}+\sqrt{1-\frac{b}{r}}\right) \\
& =-\frac{\sigma \rho_{0}\left(\frac{r_{0}}{r}\right)^{\sigma}}{1-\omega \rho_{0}\left(\frac{r_{0}}{r}\right)^{\sigma}}[r(1+\omega T)+\omega] .
\end{aligned}
$$

We plot the shape function numerically in order to study the wormhole geometry as shown in Fig. 1 fixing the initial condition as $b(1)=1$. We take some particular values of constants as $r_{0}=1, \rho_{0}=0.75, \omega=0.4$, and plot versus $r$. The plot of $b$ represents positively increasing behavior with respect to $r$. The trajectory of $1-\frac{b}{r}$ describes the positive behavior for $r \leq 1$, which meets the condition $b<r$. Figure 2 shows the null energy condition taking both components of pressure along with chosen energy density. The black curve represents $\rho+p_{\mathrm{r}}$ and blue curve describes $\rho+p_{\mathrm{t}}$ versus $r$ taking into account Eqs. (23)-(25). We see that the null energy condition holds in this case. Thus the possibility of wormhole solutions for which the normal matter satisfying the null energy condition in the background of torsion-matter coupling exists.

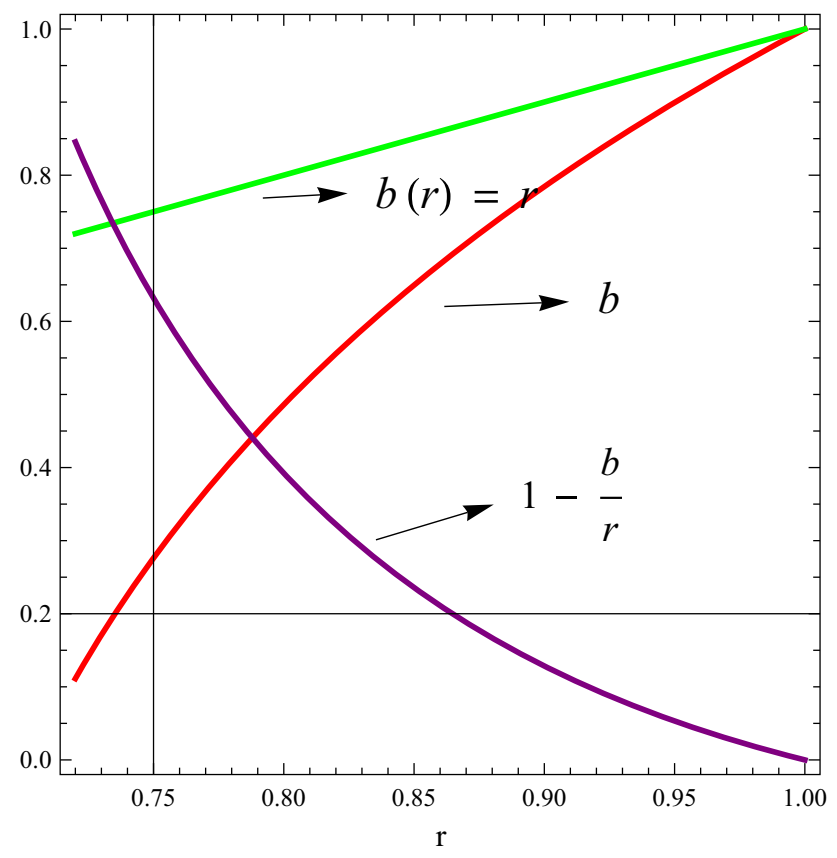

Fig. 1 Plots of the shape function $b, 1-\frac{b}{r}, b=r$ versus $r$ for Model 1 using $r_{0}=1, \rho_{0}=0.75, \sigma=3$ and $\omega=0.4$ 


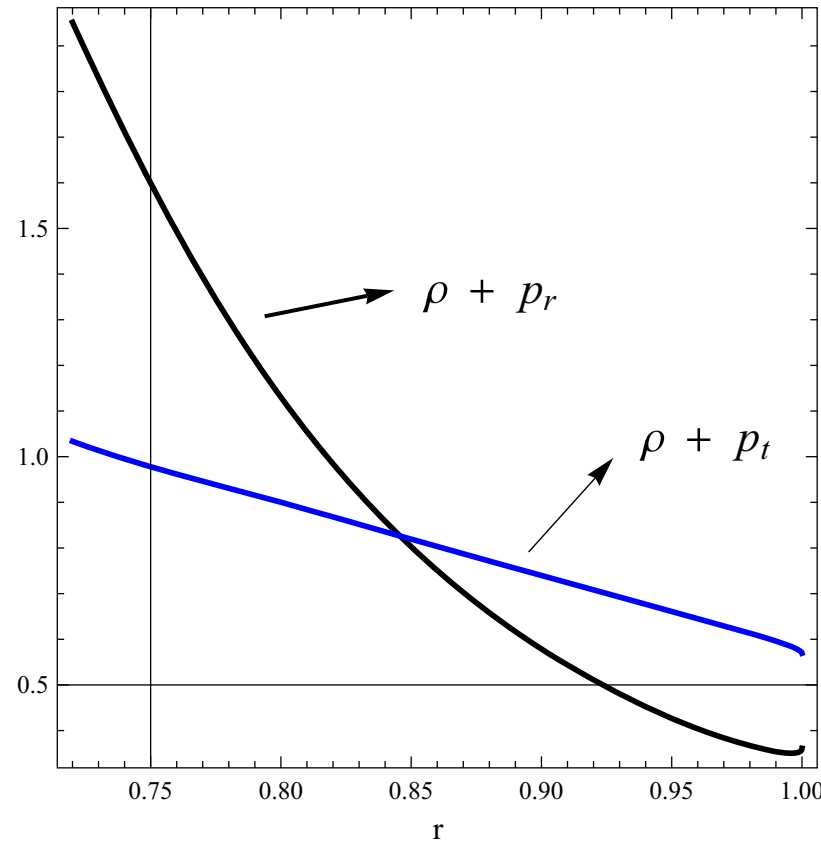

Fig. 2 Plots of the null energy condition $\rho+p_{\mathrm{r}}, \rho+p_{\mathrm{t}}$ versus $r$ for Model 1 using $r_{0}=1, \rho_{0}=0.75, \sigma=3$ and $\omega=0.4$

Figure 3 represents the general relativistic deviation profile of the null energy condition for the radial pressure component which gives the range of the coupling parameter. At the throat, the null energy condition implies

$\left.\left(\rho+p_{\mathrm{r}}\right)\right|_{r=r_{0}}=\rho_{0}-\frac{1-\omega \rho_{0}}{r_{0}^{2}+2 \omega}$.

For the chosen values of parameters, we obtain the range of the coupling constant as $\omega \geq 0.11$ for which the null energy condition holds. This describes the increasing value of the coupling constant minimizes or vanishes for the violation of the null energy condition through matter. For the case when there is no coupling, i.e., $\omega=0$, Eq. (22) reduces to $b^{\prime}=-\sigma \rho_{0} r_{0}^{\sigma} r^{1-\sigma}$. The solution of this equation is $b=$ $-\frac{\sigma \rho_{0} r_{0}^{\sigma}}{2-\sigma} r^{2-\sigma}+c$, where $c$ is an integration constant and can be determined by $b\left(r_{0}\right)=r_{0}$. After applying this condition, finally the shape function becomes

$b(r)=r_{0}\left(1+\frac{\sigma \rho_{0} r_{0}}{2-\sigma}\right)-\frac{\sigma \rho_{0} r_{0}^{\sigma}}{2-\sigma} r^{2-\sigma}$.

This shape function shows an asymptomatically flat geometry as $\frac{b}{r} \rightarrow 0$ as $r \rightarrow \infty$. Taking the same values of parameters, this function gives $b=-1.25+\frac{2.25}{r}$ representing decreasing but positive behavior for $r<1.8$ while $1-\frac{b}{r}$ holds for two sets of ranges $(r>-2.25$ and $r>1)$ or $(r<-2.25$ and $r<1)$. Also, the condition $b^{\prime}\left(r_{0}\right)<1$ meets as $b^{\prime}=-\sigma \rho_{0} r_{0}<1$. The expression $\rho+p_{\mathrm{r}}=\frac{1}{r^{4}}(2 r-2.25)$

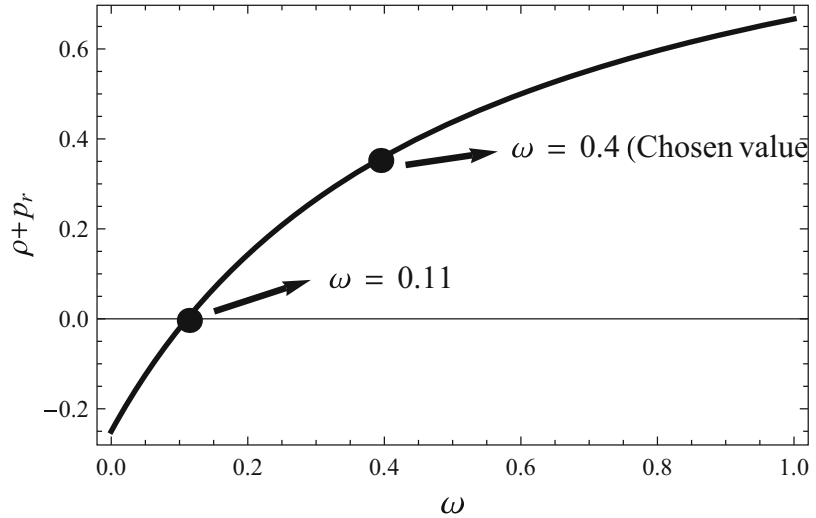

Fig. 3 The general relativistic deviation profile versus $\omega$ for Model 1 using $r_{0}=1, \rho_{0}=0.75$, and $\sigma=3$

leads to $r>1.125$ to meet the null energy condition, while $\rho+p_{\mathrm{t}}=\frac{1}{2 r^{4}}(0.25 r+4.5)$, which remains positive.

\subsection{Model 2}

As the second choice for the model, we consider the viable model with quadratic torsion term as [59]

$f_{1}=-\Lambda, \quad f_{2}=\mu T+\nu T^{2}$,

where $\Lambda>0, \mu$ and $v$ are constants. This model describes a well-known result in cosmological scenario, i.e., a matterdominated phase followed by a phantom phase of the universe. Substituting Eqs. (25) and (27) in (22), we obtain the following differential equation:

$$
\begin{aligned}
& b^{\prime}+\frac{r}{\mu+2 \nu T}\left\{\frac{\sigma(\mu+2 \nu T)}{r}-2 v T^{\prime}\right\} \\
& \times\left(\frac{b}{r}+\sqrt{1-\frac{b}{r}}-1\right)=-\frac{r^{2}}{1+\omega(\mu+2 v T)} \\
& \quad \times(1+\omega(\mu+v T) T) .
\end{aligned}
$$

Figure 4 represents the plots of the shape function under different conditions through numerical computations. The trajectory of $b$ describes positively increasing behavior for $r \geq 1$ and then decreasing behavior. The plot of $1-\frac{b}{r}$ respects positive behavior. Thus, for model 2, we obtain wormhole geometry for the torsion-matter coupling. The null energy condition for this model is plotted in Fig. 5, which shows the positivity of the condition. In order to check the relativistic deviation profile taking into account the null energy condition for the radial pressure component at the throat, we find the equation as follows:

$\left.\left(\rho+p_{\mathrm{r}}\right)\right|_{r=r_{0}}=\rho_{0}+\frac{\omega\left(\mu r_{0}^{2}+4 v\right) \rho_{0}}{r_{0}^{4}+2 \omega\left(\mu r_{0}^{2}+2 v\right)}$. 


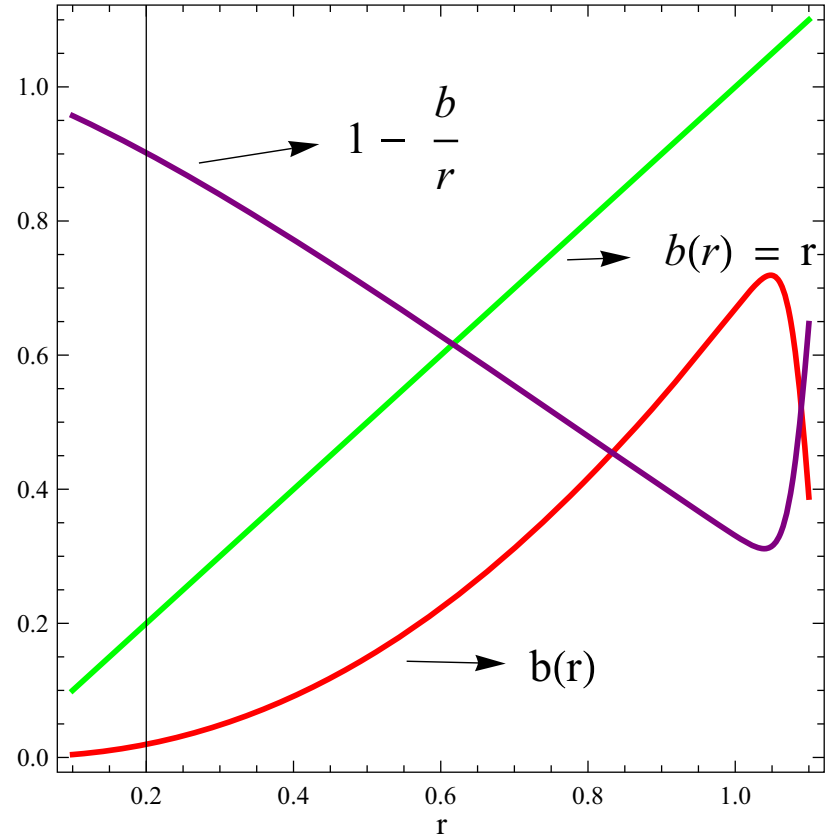

Fig. 4 Plots of the shape function $b, 1-\frac{b}{r}, b=r$ versus $r$ for Model 2 using $r_{0}=1, \rho_{0}=0.75, \sigma=3, \mu=0.2, v=-2$, and $\omega=0.4$

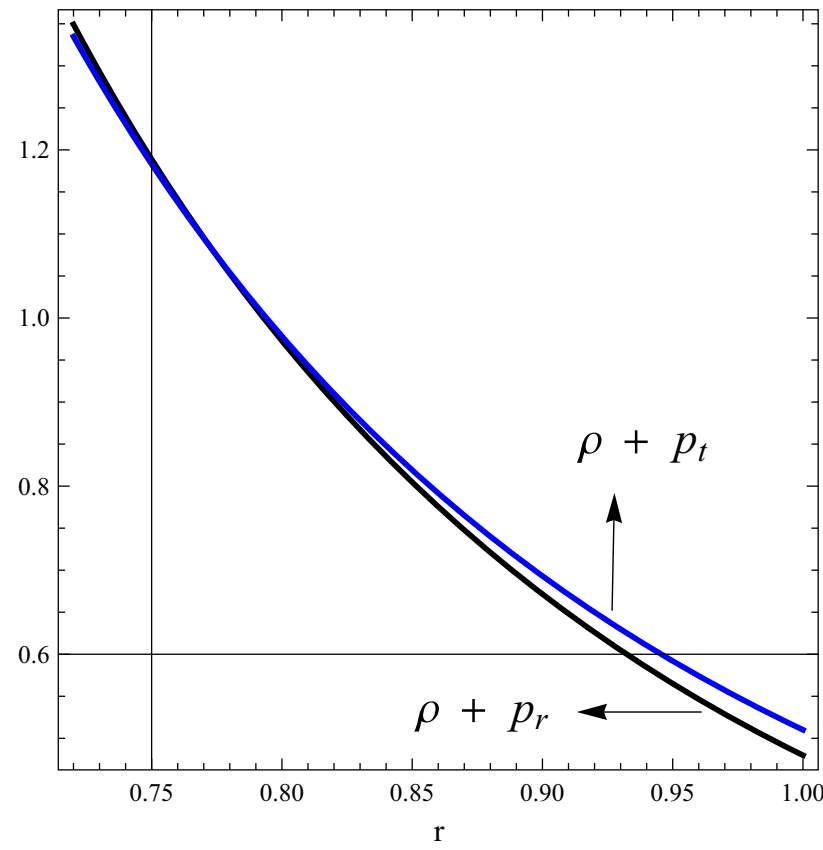

Fig. 5 Plots of the null energy condition $\rho+p_{\mathrm{r}}, \rho+p_{\mathrm{t}}$ versus $r$ for Model 2 using $r_{0}=1, \rho_{0}=0.75, \sigma=3, \mu=0.2, v=-2$, and $\omega=0.4$

Its plot is shown in Fig. 6, representing $\omega>0.15$ for the range where the null energy condition holds. This shows that the increasing value of the coupling constant minimizes or is vanishing on the violation of the null energy condition through matter. Also, we may discuss the case of zero coupling in a similar way to Model 1.

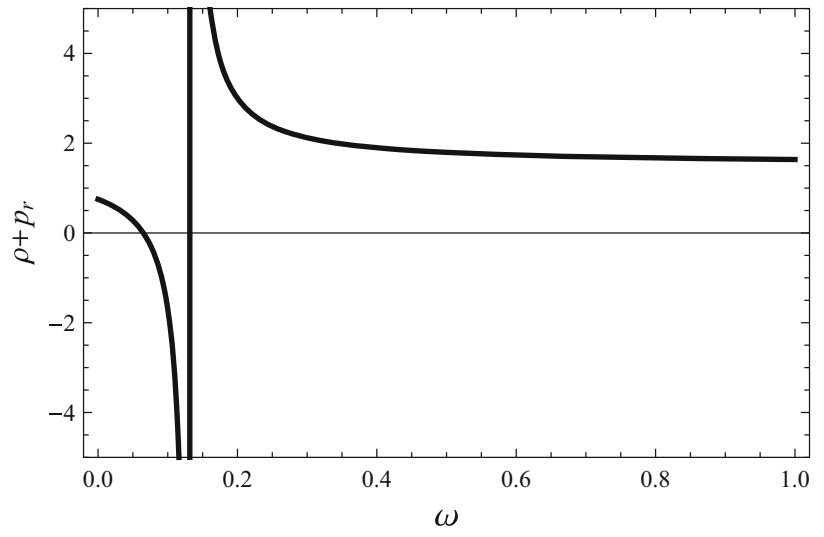

Fig. 6 The general relativistic deviation profile versus $\omega$ for Model 2 using $r_{0}=1, \rho_{0}=0.75, \sigma=3, \mu=0.2, v=-2$

\section{Concluding remarks}

The search for wormhole solutions satisfying energy conditions becomes the most interesting configuration now-a-days. Wormhole is of a tube-like shape or tunnel, which is assumed to be a source to link distant regions in the universe. The most amazing thing is the possibility of two-way travel through the wormhole tunnel which happened when the throat remains open. That is, to prevent the wormhole from collapsing at a non-zero minimum value of the radial coordinate. In order to keep the throat open, the exotic matter is used, which violates the null energy condition and we elaborated the wormhole trajectories as hypothetical paths. In order to find some realistic sources which support the wormhole geometry, our concentration goes toward modified theories of gravity. In these theories, effective scenario gives the violation of the null energy condition and the matter source supports the wormholes. In this paper, the wormhole geometries are explored taking a non-minimal coupling between torsion and matter part in extended teleparallel gravity. This coupling expresses the exchange of energy and momentum between both parts, of torsion and matter.

The extension of $f(T)$ gravity appeared in terms of two arbitrary functions $f_{1}(T)$ and $f_{2}(T)$ where $f_{1}$ is the extension of geometric part while $f_{2}$ is coupled with matter Lagrangian part through some coupling constant. At the throat, the general conditions imposed by the null energy condition taking the energy-momentum tensor of the matter Lagrangian are presented in terms of a non-minimal torsionmatter coupling. The field equations appeared in non-linear forms, which are difficult to solve for analytical solutions. Presenting various strategies to solve these equations, we have adopted to assume two viable models with a nonmonotonically decreasing function of energy density. These models involving a linear torsion scalar coupled with $T$ matter and quadratic torsion term with matter represented wormhole geometry. For these solutions, the null energy con- 
dition is satisfied. It is concluded that the null energy condition is satisfied for increasing values of coupling constant. This showed that the usage of exotic matter can be reduced or is vanishing with the higher values of the coupling constant. Thus, through the torsion-matter coupling, we have obtained some wormhole solutions in a realistic way such that the matter source satisfied the energy conditions while effective part having torsion-matter coupled terms provided the necessary violation. Finally, we remark here that this work may be a useful contribution for the present theory as well as astrophysical aspects.

Open Access This article is distributed under the terms of the Creative Commons Attribution 4.0 International License (http://creativecomm ons.org/licenses/by/4.0/), which permits unrestricted use, distribution, and reproduction in any medium, provided you give appropriate credit to the original author(s) and the source, provide a link to the Creative Commons license, and indicate if changes were made. Funded by SCOAP ${ }^{3}$.

\section{References}

1. L. Flamm, Phys. Z. 17, 448 (1916)

2. A. Einstein, N. Rosen, Phys. Rev. 48, 73 (1935)

3. M.S. Morris, K.S. Thorne, Am. J. Phys. 56, 395 (1988)

4. M.S. Thorne, K.S. Thorne, U. Yurtsever, Phys. Rev. Lett. 61, 1446 (1988)

5. F.S.N. Lobo, Phys. Rev. D 71, 084011 (2005)

6. S.V. Sushkov, Phys. Rev. D 71, 043520 (2005)

7. M. Sharif, A. Jawad, Eur. Phys. J. Plus 129, 15 (2014)

8. A. Das, S. Kar, Class. Quantum Gravity 22, 3045 (2005)

9. F.S.N. Lobo, Phys. Rev. D 73, 064028 (2006)

10. N.M. Garcia, F.S.N. Lobo, M. Visser, Phys. Rev. D 86, 044026 (2012)

11. M.G. Richarte, C. Simeone, Phys. Rev. D 76, 087502 (2007)

12. E. Teo, Phys. Rev. D 58, 024014 (1998)

13. P.E. Kashargin, S.V. Sushkov, Phys. Rev. D 78, 064071 (2008)

14. S. Kar, D. Sahdev, Phys. Rev. D 53, 722 (1996)

15. A.V.B. Arellano, F.S.N. Lobo, Class. Quantum Gravity 23, 5811 (2006)

16. S.V. Sushkov, Y.-Z. Zhang, Phys. Rev. D 77, 024042 (2008)

17. F.S.N. Lobo, M.A. Oliveira, Phys. Rev. D 80, 104012 (2009)

18. M. Jamil et al., J. Korean Phys. Soc. 65, 917 (2014)

19. F. Rahaman et al., Int. J. Theor. Phys. 53, 1910 (2014)

20. E.V. Linder, Phys. Rev. D 81, 127301 (2010)

21. S.H. Chen, J.B. Dent, S. Dutta, E.N. Saridakis, Phys. Rev. D 83, $023508(2011)$

22. P. Wu, H.W. Yu, Phys. Lett. B 693, 415 (2010)

23. J.B. Dent, S. Dutta, E.N. Saridakis, J. Cosmol. Astropart. Phys. 01, 009 (2011)

24. R. Zheng, Q.G. Huang, J. Cosmol. Astropart. Phys. 03, 002 (2011)

25. K. Bamba, C.Q. Geng, C.C. Lee, L.W. Luo, J. Cosmol. Astropart. Phys. 01, 021 (2011)
26. Y.-F. Cai, S.-H. Chen, J.B. Dent, S. Dutta, E.N. Saridakis, Class. Quantum Gravity 28, 2150011 (2011)

27. M. Sharif, S. Rani, Mod. Phys. Lett. A 26, 1657 (2011)

28. M. Li, R.X. Miao, Y.G. Miao, J. High Energy Phys. 07, 108 (2011)

29. S. Capozziello, V.F. Cardone, H. Farajollahi, A. Ravanpak, Phys. Rev. D 84, 043527 (2011)

30. M.H. Daouda, M.E. Rodrigues, M.J.S. Houndjo, Eur. Phys. J. C 72, 1890 (2012)

31. Y.P. Wu, C.Q. Geng, Phys. Rev. D 86, 104058 (2012)

32. H. Wei, X.J. Guo, L.F. Wang, Phys. Lett. B 707, 298 (2012)

33. K. Atazadeh, F. Darabi, Eur. Phys. J. C 72, 2016 (2012)

34. K. Karami, A. Abdolmaleki, J. Cosmol. Astropart. Phys. 04, 007 (2012)

35. L. Iorio, E.N. Saridakis, Mon. Not. R. Astron. Soc. 427, 1555 (2012)

36. J. Amoros, J. de Haro, S.D. Odintsov, Phys. Rev. D 87, 104037 (2013)

37. K. Bamba, S. Capozziello, M. De Laurentis, S. Nojiri, D. SezGmez, Phys. Lett. B 727, 194 (2013)

38. A. Paliathanasis, S. Basilakos, E.N. Saridakis, S. Capozziello, K. Atazadeh, F. Darabi, M. Tsamparlis, Phys. Rev. D 89, 104042 (2014)

39. G.R. Bengochea, Phys. Lett. B 695, 405 (2011); Wang, T. Phys. Rev. D 84, 024042 (2011)

40. T. Wang, Phys. Rev. D 84, 024042 (2011)

41. R.-X. Miao, M. Li, Y.-G. Miao, J. Cosmol. Astropart. Phys. 11, $033(2011)$

42. C.G. Boehmer, A. Mussa, N. Tamanini, Class. Quantum Gravity 28, 245020 (2011)

43. M.H. Daouda, M.E. Rodrigues, M.J.S. Houndjo, Eur. Phys. J. C 71, 1817 (2011)

44. R. Ferraro, F. Fiorini, Phys. Rev. D 84, 083518 (2011)

45. S. Capozziello, P.A. Gonzalez, E.N. Saridakis, Y.J. Vasquez, High Energy Phys. 02, 039 (2013)

46. K. Bamba, S. Nojiri, S.D. Odintsov, Phys. Lett. B 731, 257 (2014)

47. C.G. Böhmer, T. Harko, F.S.N. nad Lobo, Phys. Rev. D 85, 044033 (2012)

48. M. Jamil, D. Momeni, R. Myrzakulov, Eur. Phys. J. C 73, 2267 (2013)

49. M. Sharif, S. Rani, Phys. Rev. D 88, 123501 (2013)

50. M. Sharif, S. Rani, Gen. Relativ. Gravity 45, 2389 (2013)

51. M. Sharif, S. Rani, Mod. Phys. Lett. A 29, 1450137 (2014)

52. M. Sharif, S. Rani, Eur. Phys. J. Plus 129, 237 (2014)

53. M. Sharif, S. Rani, Adv. High Energ. Phys. 2014, 691497 (2014)

54. A. Jawad, S. Rani, Eur. Phys. J. C 75, 173 (2015)

55. A. Jawad, S. Rani, Adv. High Energ. Phys. 2016, 7815242 (2016)

56. O. Bertolami, C.G. Böhmer, T. Harko, F.S.N. Lobo, Phys. Rev. D 75, 104016(2007)

57. T. Harko, Phys. Lett. B 669, 376 (2008)

58. T. Harko, F.S.N. Lobo, M.K. Mak, S.V. Sushkov, Phys. Rev. D 87, 067504 (2013)

59. T. Harko, F.S.N. Lobo, G. Otalora, E.N. Saridakis, Phys. Rev. D 89, 124036 (2014)

60. G.G.L. Nashed, Astrophys. Space Sci. 357, 157 (2015)

61. C.-J. Feng, F.-F. Ge, X.-Z. Li, R.-H. Lin, X.-H. Zhai, Phys. Rev. D 92, 104038 (2015)

62. S. Carloni, F.S.N. Lobo, G. Otalora, E.N. Saridakis, Phys. Rev. D 93, 024034 (2016)

63. N.M. Garcia, F.S.N. Lobo, Phys. Rev. D 82, 104018 (2010) 